\title{
BUCHBESPRECHUNGEN
}

\section{Jeanine Bucherer}

Die Vereinbarkeit von Militärgerichten mit dem Recht auf ein faires Verfahren gemäß Art. 6 Abs. 1 EMRK, Art. 8 Abs. 1 AMRK und Art. 14 Abs. 1 des UN-Paktes über bürgerliche und politische Rechte

Beiträge zum ausländischen öffentlichen Recht und Völkerrecht, Bd. 180

Springer-Verlag, Berlin / Heidelberg / New York, 2005, 307 S., ISBN 3-540-28106-1

95,00 EUR

Die Schrift, eine von Juliane Kokott betreute Düsseldorfer Dissertation, beschäftigt sich mit einer - nicht nur, aber insbesondere - in Lateinamerika brennenden Frage des Menschenrechtsschutzes: mit der Existenz von Militärgerichten und ihrer Vereinbarkeit mit dem regional und universell verbürgten Schutz des Einzelnen auf ein faires Verfahren. Es ist ein Problemfeld, auf das die Verfasserin während ihrer Wahlstation in Peru aufmerksam wurde - ein Hintergrund, der erklärt, dass diese Schrift nie den Praxisbezug aus den Augen verliert, etwa wenn Bucherer im Kontext des in der Praxis relevanten Problems der Straflosigkeit von Militärangehörigen für Menschenrechtsverletzungen, wie das „Verschwindenlassen“, eine militärgerichtliche Zuständigkeit als ausgeschlossen nachweist. Bucherer untersucht in ihrer Arbeit die Vereinbarkeit von Militärgerichten mit dem Recht auf ein faires Verfahren gemäß Art. 14 Abs. 1 IPBPR, Art. 8 Abs. 1 AMRK und Art. 6 Abs. 1 EMRK. Im Mittelpunkt dieser rechtsvergleichenden Prüfung stehen der Inhalt und die Auslegung der Vorschriften durch das jeweils vertraglich zuständige Kontrollorgan.

Einführend beginnt die Schrift mit einem informativen Überblick über die geschichtliche Entwicklung von Militärgerichten, die etwa das noch immer bestehende Merkmal der absoluten Verantwortung des Befehlshabers offenbart, und stellt an Beispielen die beiden Modelle dieser Gerichtsbarkeit vor - solche, die im Einzelfall einberufen werden, gegenüber denen, die als ständige Gerichte in das Rechtssystem eingegliedert sind. Darauf aufbauend, zeigt die Verfasserin Entwicklungstendenzen der nationalen Militärgerichtsbarkeiten auf - im lateinamerikanischen Raum hin zu einer Einbettung in den verfassungsrechtlichen Rahmen der Justiz -, bevor sie sich mit der zentralen Frage nach der Rechtfertigung für die Existenz von Militärgerichten auseinandersetzt; hierbei weist Bucherer zu Recht auf eine rechtsstaatlich gefährliche Vermengung der Argumente hin, wenn aus der repressiven Funktion der Streitkräfte auf ihre Kompetenz auch zur richterlichen Funktion geschlossen wird.

Ab Kapitel 2 (S. 25 ff.) wendet sich Bucherer den konkreten Verträgen und Vertragsvorschriften zu, die zum Prüfungsmaßstab gewählt wurden und klärt zunächst, dass Militärge- 
richte den Gerichtsbegriff aller drei Verträge erfüllen, wobei in der Spruchpraxis der Organe dies unter dem Aspekt der Unabhängigkeit und der Unparteilichkeit der Militärrichter problematisiert wird. Folgerichtig untersucht die Autorin anschließend umfänglich die Spruchpraxis zu diesen beiden Tatbestandsmerkmalen. Insgesamt diagnostiziert Bucherer ,ähnliche Bedenken“ der Organe, etwa zur Ernennung und Disziplin der Richter sowie zur Beteiligung von Militärangehörigen an Strafverfahren. Zu militärgerichtlichen Strafverfahren gegen Zivilpersonen macht Bucherer - anders als beim Menschenrechtsausschuss - für den EGMR und die frühere EKMR, die angesichts der Beschwerdezahlen wie so häufig im Menschenrechtsschutz das meiste und differenzierteste Entscheidungsmaterial liefern, eine - ihrer Meinung nach zu starke - Orientierung an den Merkmalen der Unabhängigkeit und der Unparteilichkeit und damit äußerer Umstände aus; insbesondere bewertet sie das bei den europäischen Organen festzustellende Ausweichen auf ein zusätzliches Kriterium, „den äußeren Anschein“, als unrichtig - ein Fazit, das allerdings in der Gesamtbewertung nicht mehr so deutlich wird (S. 101 f.). Vor allem die seitens der Interamerikanischen Menschenrechtskommission ausgemachte Weiterentwicklung hin zu einem grundsätzlichen Verbot von Verfahren gegen Zivilpersonen vor Militärgerichten anhand eines neuen Kriteriums, des „,natural judge“, findet die Zustimmung der Autorin, da es „um etwas wie das internationale Recht auf den gesetzlichen Richter" (S. 102) gehe.

Die Frage, wie sich ein solches Recht dogmatisch begründen lässt, beschäftigt die Verfasserin im Folgenden (Kapitel 4, S. 103 ff.) - dem Herzstück ihrer Arbeit, das eigene Forschungsergebnisse präsentiert. Sie bejaht ein solches Recht, für das die Terminologie des „zuständigen Richters“ gewählt wird und das eine Zuständigkeit der Militärgerichtsbarkeit für Zivilisten ausschließt: Für den UN-Pakt und die AMRK stützt sie sich, anknüpfend an die interamerikanischen Organe, überzeugend auf eine autonome Auslegung des Merkmals Zuständigkeit mit Hilfe des Willkürverbots und des Rechtsstaatsprinzips; für die EMRK liest Bucherer, wiederum gestützt u.a. auf das Gebot der Rechtsstaatlichkeit, in nachvollziehbarer Weise das „Recht auf ein zuständiges Gericht“ als ungeschriebenes Tatbestandsmerkmal in Art. 6 Abs. 1 EMRK hinein.

Ein Überblick über die Situation von Militärgerichten in Ausnahmezuständen, in denen solche Gerichte besonders gerne eingesetzt werden, runden Bucherers Untersuchung ab; für die Autorin ist auch durch eine Derogation vom Recht auf ein faires Verfahren die strafrechtliche Verfolgung von Zivilpersonen durch Militärgerichte nicht zu rechtfertigen.

Die von Bucherer gewählte Thematik zeigt nicht zuletzt auch, dass es sich bei der Einrichtung von Militärgerichten um eine aktuelle Problematik handelt, vor der auch vermeintlich gefestigte Demokratien nicht gefeit sind, wie die strafrechtliche Zuständigkeit von Militärgerichten für ausländische Terrorismusverdächtige in den USA als Reaktion auf die Anschläge des 11. September 2001 offenbart. In einem interessanten „Nachtrag“ beschäftigt sich die Autorin mit dieser Entwicklung und analysiert, ob die zur Verfolgung der Taliban und Al Quaida-Kämpfer eingerichteten Militärkommissionen mit dem Recht auf ein faires Verfahren gemäß Art. 14 IPBPR vereinbar sind. In gewohnt klarer, sachlicher und einleuchtender Abhandlung verneint Bucherer dies, ebenso wie sie zu Recht Verstöße 
gegen die Genfer Konventionen bejaht, die bei der Auslegung des Paktes zu berücksichtigen sind, etwa durch die Behauptung, bei den Talibankämpfern handele es sich nicht um Kriegsgefangene im Sinne der Konventionen, sondern um ,unlawful combatants“.

Kurz: In einem knappen, problemorientierten und konzentrierten Stil führt Bucherer in ihrer gut lesbaren Arbeit durch die Problematik - eine Problematik, die sie übrigens den Teilnehmern der Jahrestagung des Arbeitskreises für Überseeische Verfassungsvergleichung (in Mainz, 24.-26. Juni 2005) bereits profunde in Ausschnitten vermittelte. Die Autorin hat eine rechtsvergleichende Analyse vorgelegt, die auch gerade aufgrund der eigenen Lösungsvorschläge zum „Recht auf ein zuständiges Gericht“ einen wertvollen Beitrag zur Frage der Vereinbarkeit von Militärgerichten mit Internationalem Recht und zu ihrer Unzuständigkeit für die Verfolgung von Zivilpersonen leistet.

Michaela Wittinger, Karlsruhe

\section{Ingo Malcher}

\section{Der Mercosur in der Weltökonomie}

Eine periphere Handelsgemeinschaft in der neoliberalen Globalisierung

Nomos Verlagsgesellschaft, Baden-Baden, 2005, 279 S. (Nomos Universitätsschriften

Politik Bd. 129) zugl. Diss. Marburg 2004; 51,00 EUR; ISBN 3-8329-1266-5

Im Vergleich mit dem mainstream der Mercosur-Publikationen erscheint dieses Buch gegen den Strich geschrieben. Der Autor geht bei seiner Analyse von der „Kritischen Theorie der Internationalen Beziehungen“ aus und vertritt damit eine dezidiert neomarxistische Position. Grundlage ist die politische Theorie des legendären italienischen Kommunistenführers Antonio Gramsci, die im einleitenden Kapitel dargestellt wird. Danach beruht der Staat auf der Zivilgesellschaft und bildet mit dieser eine Einheit. Die Herrschaft über beide wird nicht allein durch Zwang aufrechterhalten. Ein zentraler Begriff ist vielmehr die Hegemonie, worunter die intellektuelle Führung zu verstehen ist, die durch den Konsens der Beherrschten gefestigt wird. Der Konsens wird durch Zugeständnisse an die beherrschte Klasse erreicht: „Das System wird dadurch stabilisiert, weil das Leben für die beherrschten Klassen angenehmer wird“ (S. 19). Dieses Gesellschaftsmodell wird nun im Anschluss an den Kanadier Robert Cox, den früheren Direktor des International Institute of Labour Studies der ILO, auf die internationale Ebene übertragen. Hegemoniale Ordnung stützt sich danach nicht allein auf ökonomische oder militärische Vorherrschaft, sondern bedarf einer führenden Ideologie, die durch internationale Institutionen abgesichert und von den Schwächeren als Ausdruck eines allgemeinen Interesses akzeptiert wird. Als eine solche Ideologie versteht der Autor den Neoliberalismus, zu dessen politischem Programm die Globalisierung gehört. Entgegen verbreiteter Meinung führt diese nicht zum Bedeutungs- 
verlust des Nationalstaates, sondern wird umgekehrt durch dessen Vermittlerrolle erst ermöglicht.

Auf dieser Grundlage zeichnet der Autor zunächst ein Bild der neoliberalen „Gegenrevolution“ (M. Friedman) als Gegenentwurf zum Keynesianismus und ihrer Wirkung in Lateinamerika. Hier war das von der CEPAL propagierte Modell der Importsubstitution mit der Schuldenkrise der achtziger Jahre fragwürdig geworden, wobei der Autor allerdings einen ursächlichen Zusammenhang verneint. Demgegenüber hatte bereits seit 1973 ein neoliberales Experiment unter den Laborbedingungen der chilenischen Diktatur stattgefunden, in klarem Widerspruch zu der von Friedman selbst postulierten immanenten Verbindung von wirtschaftlicher und politischer Freiheit. In den neunziger Jahren wurde dann überall in Lateinamerika die Wende vollzogen: Das neoliberale Manifest des „Washington Consensus" (Williamson 1990) wurde bestimmend für die Wirtschaftspolitik der lateinamerikanischen Regierungen. Während diese Politik vor allem im Bereich der Inflationsbekämpfung erfolgreich war, führte sie auf der anderen Seite zu einer Verschärfung der sozialen Probleme und zu einem weiteren Anstieg der Auslandsschuld und damit zur Abhängigkeit der nationalen Finanz- und Wirtschaftspolitik von ausländischen Rating-Agenturen. Einen erneuten Wendepunkt sieht der Autor in der Zahlungskrise Argentiniens zur Jahreswende 2001/02: Nachdem das Land jahrelang als Musterschüler des IWF alle neoliberalen Reformen umgesetzt hatte, geriet es nach dem Ende der Privatisierungen in die Rezession und schließlich in die Zahlungsunfähigkeit - für den Autor die konsequente Folge der neoliberalen Politik, während andere umgekehrt gerade die fehlende Konsequenz bei der Umsetzung dieser Politik dafür verantwortlich machen (H. Köhler).

Komplementär zur Globalisierung findet eine Regionalisierung des Welthandels statt. Für die Länder der Peripherie ist die Herausbildung von Regionalblöcken eine Möglichkeit, sich in den Weltmarkt zu integrieren, sie unterliegen aber dabei einem erhöhten Anpassungsdruck. In diesem Sinne beschreibt der Autor den Mercosur der neunziger Jahre als „neoliberales Regionalprojekt“ (S. 120), das im Gegensatz zur früheren argentinisch-brasilianischen Integration keine eigenständige regionale Entwicklungsstrategie verfolgte, sondern vielmehr im Zeichen des sog. Offenen Regionalismus als Sprungbrett in den Weltmarkt konzipiert war. Wenn er in diesem Zusammenhang ein Demokratiedefizit beklagt, weil die nationalen Parlamente den Vertrag von Asunción nur annehmen oder ablehnen, aber nicht verändern konnten (S. 122), so ist dem freilich entgegenzuhalten, dass dies auch sonst (mit Ausnahme der USA) der internationalen Übung beim Abschluss von Staatsverträgen entspricht. Umgekehrt ist gerade zu betonen, dass den Mercosur-Normen im Gegensatz zum EG-Recht keine unmittelbare Wirkung zukommt, so dass es zu ihrer Durchsetzung vielfach noch der legislativen Umsetzung in den beteiligten Staaten bedarf. Auch die institutionelle Struktur des Mercosur wird auf S. $119 \mathrm{f}$. sehr unvollständig beschrieben; so wird die wichtige Rolle der Handelskommission als Organ des Mercosur völlig übersehen und diese offenbar mit einer der zahlreichen Unterarbeitsgruppen verwechselt. Dem Autor kommt es allerdings mehr darauf an, die wirtschaftliche Entwicklung des Mercosur mit ihren Krisen und Konflikten nachzuzeichnen (ohne dabei die in 
diesem Zusammenhang entwickelte Schiedspraxis des Mercosur auch nur zu erwähnen). Die Zahlungskrise Argentiniens wertet er als Ausdruck der Krise der neoliberalen Hegemonie in Südamerika und zugleich als Chance für ein gewandeltes Mercosur-Projekt im Sinne eines „pragmatischen neo-keynesianischen Realismus“ (S. 143). Ansätze für eine solche politische Alternative sieht er in den linksgerichteten Regierungen von Kirchner in Argentinien und Lula in Brasilien und der von ihnen gemeinsam vorangetriebenen Wiederbelebung des Mercosur (S. 159: „Integration statt Revolution“) - wobei er freilich die Aussichten für eine Überwindung der bestehenden neoliberalen Strukturen durchaus skeptisch einschätzt.

Auf der internationalen Ebene findet sich der Mercosur im Spannungsfeld zwischen den großen wirtschaftlichen Blöcken der USA und der EU, von deren „Ringen um die Vorherrschaft in Lateinamerika“ das letzte Drittel des Buches handelt. Mit etwas krauser Logik sieht der Autor in der Annäherung zwischen der EU und dem Mercosur den Auslöser für die 1990 (!) von den USA gestartete „Americas Initiative“ (S. 162). Die daraus folgenden Verhandlungen über eine panamerikanische Freihandelszone (FTAA/ALCA) werden dagegen sehr sorgfältig und detailliert beschrieben, auch wird der Modellcharakter des zwischen den USA und Chile 2003 unterzeichneten Freihandelsabkommens zutreffend betont (das Datum der Unterzeichnung freilich zunächst auf 2002, später auf 2004 verlegt, S. 153 und 160). Dem wird das Bemühen der Europäischen Gemeinschaft gegenübergestellt, auf der Grundlage des 1995 geschlossenen und 1999 in Kraft getretenen Rahmenabkommens mit dem Mercosur das dort anvisierte Ziel einer ,,interregionalen Assoziation“ im Verhandlungswege zu konkretisieren und zugleich durch die Freihandelsvereinbarungen mit Mexiko von 1997/2000 (S. 216 ungenau auf 1999 datiert) in den unmittelbaren Einflussbereich der USA einzudringen. Das Assoziationsabkommen der EG mit Chile von 2002 (in Kraft seit 2003) wird dagegen in diesem Zusammenhang nicht erwähnt. Die ausführliche Darstellung der Verhandlungen der USA bzw. der EG mit dem Mercosur zeigt deutliche Parallelen auf, insbesondere was die strittige Frage der Agrarsubventionen der Industriestaaten angeht. Ähnlich ist auch die Rolle des „American Business Forum“ (mit unterschiedlichen Gründungsdaten S. 174, 186) und des „Mercosur European Union Business Forum“ als Vertretung der transnationalen Großunternehmen im jeweiligen Verhandlungsprozess, während kleinere und mittlere Unternehmen sowie die übrigen Sektoren der Zivilgesellschaft (z.B. Gewerkschaften) faktisch davon ausgeschlossen bleiben. Für den Autor handelt es sich dabei letztlich um den Wettstreit zweier hegemonialer Strategien, die für den Mercosur in jedem Fall eine Festschreibung der Einbindung in die neoliberale Globalisierung bedeuten würden. Ob dieser tatsächlich in der Lage ist, sich dem zu entziehen und ein alternatives Entwicklungsmodell im Sinne einer „Gegenhegemonie“ durchzusetzen, hält er angesichts der Zahlungsunfähigkeit Argentiniens und der hohen Verschuldung Brasiliens selbst für unsicher.

Auch wer den theoretischen Ansatz des Autors nicht teilt, wird in diesem Buch eine anregende Lektüre finden. Obwohl der Text von einem klaren politischen Standpunkt geprägt wird, ist er doch niemals dogmatisch und auch offen für abweichende Interpretationen. 
Derzeit arbeitet der Autor in Argentinien als Korrespondent für deutschsprachige Zeitungen, was den teilweise essayistischen Charakter des Buches erklären mag. Immerhin handelt es sich dabei um seine gekürzte Marburger politologische Dissertation, wobei die Kürzung offenbar für viele fehlgehende Verweisungen verantwortlich ist. Der Text enthält trotz der Kürzung noch immer zahlreiche Redundanzen, was bis zur wörtlichen Wiederholung ganzer Passagen geht (vgl. etwa S. 43, 49, 82 oder 164, 173); darunter leidet bisweilen die Stringenz der Darstellung. Ein simples Rechtschreibprogramm hätte viele Druckfehler eliminieren können, ärgerlich sind auch Flüchtigkeiten wie Michael (!) statt Milton Friedman (S. 28), korporative statt komparative Kostenvorteile (S. 147) oder die mehrfach so bezeichnete „Aerea de Libre Comercio“ (S. 164, 174). Wer aber aus der täglichen Zeitungslektüre gewohnt ist, dass aktualitätsbezogene Texte heute in der Regel nicht mehr sorgfältig redigiert werden, wird dies dem Autor nicht allzu schwer anlasten. Zu bemängeln ist dagegen das rudimentäre Abkürzungsverzeichnis.

post scriptum: Seit dem Zeitpunkt, in dem das Manuskript des vorliegenden Buches abgeschlossen wurde, sind in Lateinamerika Veränderungen eingetreten, die durchaus in die vom Autor aufgezeigte Richtung gehen. Mit den Wahlsiegen von Tabaré Vázquez in Uruguay, von Evo Morales in Bolivien und von Michelle Bachelet in Chile zeichnet sich ein deutlicher Linksruck ab. Argentinien und Brasilien haben ihre wirtschaftspolitische Handlungsfreiheit in Teilen wiedergewonnen, nachdem Argentinien die Umschuldung bewältigt und Brasilien eine weitgehende Rückführung der Auslandsschulden erreicht hat. Im November 2005 scheiterte auf dem 4. Amerika-Gipfeltreffen in Mar del Plata das Projekt einer Amerikanischen Freihandelszone an dem geschlossenen Nein der vier MercosurStaaten und Venezuelas. Und einen Monat später wurde auf der 29. Mercosur-Ratstagung in Montevideo die Aufnahme Venezuelas als Vollmitglied in die Wege geleitet. Der künftige Weg des Mercosur bleibt damit weiterhin offen.

Jürgen Samtleben, Hamburg

Christoph Moeskes (Hrsg.)

\section{Nordkorea}

Einblicke in ein rätselhaftes Land

Ch. Links Verlag, Berlin, 2004, 240 S., 15,90 EUR

Die Demokratische Volksrepublik Korea (Nordkorea) ist auch heute noch das wohl abgeschottetste und verschlossenste Land der Welt, über das zuverlässige Informationen kaum zu erlangen sind. So ist es eine dankenswerte Leistung des Herausgebers, die Schilderungen von 27 Beobachtern (26 Ausländern und einem geflohenen Nordkoreaner) aus den Jahren 1989 bis 2004 in einem handlichen Band zu vereinen. Dass viele davon zuvor in 
fürs Normalpublikum schwer zugänglichen Publikationen veröffentlicht wurden, steigert eher ihren Wert, als dass es ihn mindert.

Trotz der hier gegebenen Vergleichsmöglichkeit sind durch die Lektüre wohl Einblicke möglich, kaum aber Einsichten, wie der Herausgeber in seiner langen (28 Seiten!) Einleitung hervorhebt. Dies liegt teils an der nahezu lückenlosen Betreuung, die das Regime seinen Gästen angedeihen lässt, teils aber auch an der höchst unterschiedlichen Sichtweise der Autoren: Einige haben, wie der Herausgeber bemerkt, die „Inszenierungen“ des Regimes offenbar akzeptiert, während andere zumindest versuchten, hinter die Kulissen ebendieser Inszenierungen zu schauen bzw. Risse in der Fassade zu entdecken.

Ein Beispiel: Während der deutsche Experimentalphysiker Gert G. Harigel (,In der Höhle des Löwen“, 2002) „keine unterernährten, schlecht gekleideten Menschen“ sah und das „köstliche und reichliche“ Hotel-Essen rühmt, fielen dem polnischen Politologen Marceli Burdelski („Überleben im Staatskult“, 2003) bei seinem etwa gleichzeitigen Aufenthalt überall ,überladene Lastwagen, voll mit erschöpften, schlecht aussehenden Menschen“ auf. Harigel bemerkt allerdings auch, er habe vor seiner Reise ,weder eine Genehmigung noch Ratschläge von irgend jemandem" benötigt...

Vergleicht man die Beobachtungen in ihrer zeitlichen Abfolge - die Beiträge sind auch in den einzelnen Abschnitten nicht chronologisch geordnet -, so ist eine zaghafte Lockerung der Abschottung und Vergrößerung der den Besuchern gewährten Freiräume nicht zu übersehen, die allerdings weit hinter dem zurückbleibt, was in anderen kommunistisch gebliebenen Ländern wie Vietnam inzwischen gängige Praxis ist. Dies gilt auch nach den als Zäsur und Beschleuniger empfundenen Wirtschaftsreformen von 2002, die nicht als „Reformen“ bezeichnet werden dürfen: Mit dem Vorsitzenden der Deutsch-Koreanischen Parlamentariergruppe im Deutschen Bundestag, Hartmut Koschyk, dessen Beitrag „Einmal gesehen ist besser als 100 mal gehört“ (2003) den Band beschließt, einigte man sich auf „,interessante Veränderungen“!

Doch was haben die Autoren beobachtet? Der Band ist in sechs Abschnitte unterschiedlichen Umfangs gegliedert, die sich zudem teilweise thematisch überlappen. Alle Beobachter waren in der Hauptstadt, und so ist der erste Abschnitt „Pjöngjang“ mit acht Beiträgen der umfangreichste. Es folgen „Inszenierungen“ mit drei und „Provinz“ mit fünf Beiträgen. Nur einen Beitrag weist der Abschnitt zum besonders geheimen Thema „Lager“ auf, während die auf Grund der desolaten Wirtschaftslage lebensnotwendige „Hilfe“ mit drei Beiträgen vertreten ist. Der zur Überwindung der Isolierung erforderliche „Austausch“ komplettiert mit sieben Beiträgen die Sammlung.

Fast alle Autoren sind beeindruckt, fasziniert, meist auch abgestoßen durch den überwältigenden Personenkult, der um den verstorbenen „Großen Führer“ und „Ewigen Staatspräsidenten“ KIM Il-sung und seinen Sohn und Erben, den „Geliebten Führer“ KIM Jong-il in zahlreichen monströsen Gedenk- und Weihestätten betrieben wird, deren Besuch obligatorisch ist. Den meisten sind auch die adrett uniformierten Polizistinnen aufgefallen, die mit ballettartig einstudierten Bewegungen an den Kreuzungen der bis zu achtspurigen Prachtstraßen Pyongyangs den nicht vorhandenen Verkehr regeln (so Rainer Stahl: „Der große 
und der kleine Führerschein, 2001; Elke Werry: „Die Blumen von Pjöngjang: Tanzende Polizistinnen“, 2002). Kontrastbild ist die nachts wegen Strommangels in völliges Dunkel versinkende Stadt (Herausgeber Moeskes: Einleitung) oder auch die Diskrepanz zwischen den zwei taghell erleuchteten für Besucher zugänglichen U-Bahn-Stationen und den düster und ungepflegt wirkenden übrigen, die allerdings nur die sprachkundige Birke Dockhorn („Abenteuer auf Schienen. Pjöngjang mit und ohne Begleitung“, 1996) zu Gesicht bekam, wenn sie ihren Begleitern vorübergehend entwischen konnte.

Ein anderes Schlaglicht: Ein Besucher wurde bei der Visa-Beantragung gebeten, gewissermaßen als Gegenleistung Unterlagen über elektronische Geräte zu besorgen (Volker Hagemeister: „Röntgen bitte. Tourismus als Staatsbesuch“, 2003)! Darauf muss man gut essen gehen, wofür Sofia Malmquist (,Essen gehen in Pjöngjang. Eine Anleitung“, 2003) nützliche Hinweise gibt.

Die bestimmenden Eindrücke aus der Provinz hat Eckart Dege 1996 in der bis heute gültigen Formel „Leere Autobahnen, heilige Gipfel“ zusammengefasst, zu ergänzen durch Abstecher an die Demarkationslinie (Werner Adam: „Kalter Krieg mit Aussicht“, 1994) und in das von Süd-Korea aus unter strenger Abschirmung von der nordkoreanischen Bevölkerung zugängliche Kumgang-Gebirge (Anne Schneppen: „Prada und Pellkartoffeln“, 2004).

Begrenzten Einblick in einen besonders düsteren Aspekt gewährt KANG Chol Hwan mit „Die heiß begehrten Kaninchen. Im Arbeitslager“ (2000): Mit neun Jahren wurde er mit seinen Eltern in Sippenhaft für ein „Vergehen“ des Großvaters in ein Straflager eingewiesen und erst 10 Jahre später nach - offenbar vergeblicher! - Umerziehung wieder freigelassen. Über China gelang ihm die Flucht nach Süd-Korea. Seine Erinnerungen wurden in New York als Buch (,The Aquariums of Pyongyang“) veröffentlicht.

Als besonders markantes Beispiel für trotz vieler Hemmnisse gelungene Hilfe sei hier nur auf Hans Strehling: „Gefrorenes Glück. Die erste deutsche Rindfleisch-Lieferung“ (2001) verwiesen: Wegen der BSE-Krise in Deutschland unverkäufliches Rindfleisch konnte zur Linderung der Hungersnot in Nord-Korea beitragen.

Auch im letzten Abschnitt „Austausch“ ist naturgemäß von deutsch-nordkoreanischen Begegnungen und Projekten die Rede, wobei viele auch aus heutiger Sicht positive Beiträge der DDR zur Sprache kommen. Eindrucksvoll ist der fast ein halbes Jahrhundert umfassende Rückblick von Helga Picht: „Rückbesinnung auf die eigenen Kräfte. Das nationale Kulturschaffen“ (2003), der in Anspielung auf die gesamtkoreanische Sangesfreude in die resignierende Empfehlung mündet, ,dass es vielleicht besser wäre, wenn die Vertreter beider Korea nicht gegeneinander reden, sondern miteinander singen würden“. Dass dieser Gedanke gar nicht so abwegig ist, beweist der darauf folgende Beitrag von Alexander Liebreich: „Pjöngjang singt, Deutschland singt mit“ (2004), der 2002 als Dirigent und 2003 als Gastprofessor in Nord-Korea war. Dies war in erster Linie den zielstrebigen Bemühungen des langjährigen Leiters des Goethe-Instituts Seoul, Uwe Schmelter (jetzt in Tokyo mit Regionalzuständigkeit für ganz Ostasien) zu verdanken und erwies sich als Türöffner für weitere Projekte wie den im Buch nur als ,geplant“ erwähnten, inzwischen 
aber eröffneten deutschen Lesesaal in Pyongyang. Da bleibt nur zu hoffen, dass der Zugang tatsächlich so „frei“ ist, wie von der nordkoreanischen Regierung zugesagt.

26 in einem zentralen Bildteil zusammengefasste Farbfotos und sieben in den Text eingestreute Schwarz-Weiß-Aufnahmen lassen die Eindrücke der Verfasser auch optisch nachvollziehen.

Was nach der Lektüre bleibt, ist der Eindruck vieler bunter Mosaiksteinchen, die sich zum Bild nicht fügen wollen, sondern allenfalls zu kaleidoskopartig wechselnden Impressionen. Eines aber ist sicher: Wer dieses Buch gelesen hat, wird sich über nichts mehr wundern, was an Nachrichten aus Nordkorea herausdringt.

Karl Leuteritz, Königswinter

\section{Sylvia Bräsel (Hrsg.)}

\section{Siegfried Genthe: "Korea - Reiseschilderungen"}

Erfurter Reihe zur Geschichte Asiens (ERGA), Band 7

iudicium Verlag, München, 2005, 404 S., ISBN 3-89129-786-6; 40,00 EUR

Ein bemerkenswertes Juwel hat Sylvia Bräsel pünktlich zum Koreajahr 2005 in der von Reinhard Zöllner herausgegebenen „Erfurter Reihe zur Geschichte Asiens (ERGA)“ präsentiert: Die 16 Reiseschilderungen, die 1901/02 der damalige Ostasienkorrespondent der „Kölnischen Zeitung“, Siegfried Genthe, während und nach einer im Anschluss an die Niederschlagung des Boxer-Aufstands in China durch westliche Truppen unternommenen Koreareise für seine Zeitung geschrieben hatte. Als Buch waren sie von seinem Studienfreund Georg Wegener 1905 im gemeinnützigen ,Allgemeinen Verlag für deutsche Literatur" in Berlin herausgegeben worden, nachdem Genthe 1904 mit knapp 34 Jahren einem Raubmord zum Opfer gefallen war. Dies in Marokko, wo der Aufenthalt für Europäer damals etwa so riskant gewesen sein muss wie heute im Irak. Nur dauerte die Nachrichtenübermittlung länger. In makaberer Parallele zu jüngeren irakischen Entführungsfällen hatte Genthe im Vertrauen auf seine arabischen Sprachkenntnisse und seine Kontaktfähigkeit die Warnungen der deutschen Auslandsvertretung nicht beachtet und als Bedenkenträgerei ängstlicher Diplomaten abgetan.

Doch zurück zu den Reiseschilderungen aus Korea! Leider hat Bräsel die Einleitung Wegeners nicht mit publiziert, dafür aber in zwei längeren eigenen Einleitungskapiteln (S. VIIXLIX) einmal Genthes Familien- und Lebensgeschichte, zum anderen die „Rezeptionsgeschichte der Reisebeschreibung 100 Jahre nach ihrer Erstveröffentlichung" nachgezeichnet. Dort weist sie mit zahlreichen Vergleichen zu anderen zeitgenössischen Reisebeschreibungen darauf hin, dass Genthe von dem damals vorherrschenden westlichen Überlegenheits- 
gefühl gegenüber Ostasien völlig frei war und die andersartige Kultur aus sich selbst heraus $\mathrm{zu}$ verstehen suchte.

In der Tat wird bei der Lektüre deutlich, dass das Reisen für Genthe ein kontinuierlicher Lernprozess war, in dessen Verlauf er sich mehrfach korrigierte: Bezeichnet er in seinen ersten Berichten bis zur Landung in „Tschemulpo“ (Chemulpo; heute Inchon) die Koreaner offenbar auf Grund von Angaben japanischer und westlicher Gesprächspartner und der von ihm konsultierten wenigen westlichen Reisebeschreibungen als unkultiviert, schmutzig, träge und aufdringlich neugierig, so ändert sich sein Urteil durch eigene Erfahrung in allen Punkten: Die Pferdetreiber seiner Karawane können die Inschriften am Wegesrand lesen, Dorfbewohner baden täglich im Fluss, Bauern und Handwerker sind unermüdlich tätig, ebenso die Arbeiter im deutschen Goldbergwerk „Tangkogä“ (Tonggogae), und hinsichtlich der Neugier erkennt er die beiderseitige „Menageriesituation“, in der das Bestreben der Einheimischen, Wesen und Beschaffenheit des Fremden zu erkunden, ebenso legitim ist wie dessen Drang, Sitten und Gebräuche eben dieser Einheimischen zu erforschen. Vollends als Geistesverwandte erscheinen ihm die Koreaner - in betontem Gegensatz zu Chinesen und Japanern! -, nachdem ihm mehrfach in unwegsamer Gegend begegnende Reisende auf die Frage nach dem Zweck ihrer Reise zur Antwort gaben: „Die Schönheit der Aussicht zu genießen!“ Seine Prognose allerdings, die Koreaner hätten bessere Aussichten, zur Spitze der zivilisierten Welt aufzuschließen als ihre Nachbarn - ,sie haben die helleren Köpfe und die weiteren Herzen“ - musste noch Jahrzehnte auf ihre Verwirklichung warten. Auch beim Ortsnamen der Hauptstadt lernt Genthe dazu: Schreibt er anfangs noch, wie damals in Deutschland üblich, „Söul“, so merkt er bald, dass der Name auf Koreanisch zwar zweisilbig geschrieben, aber einsilbig gesprochen wird, widmet dieser Tatsache einen ganzen Abschnitt und schreibt nun konsequent „Ssoul“. Manche Schreibungen sind allerdings gewöhnungsbedürftig: Den üblicherweise „Chosun“ geschriebenen Landesnamen der, wie er mehrfach betont, nicht „Land der Morgenruhe“, sondern „... der Morgenfrische“ bedeutet - schreibt er „Tschossönn“, und hinter „Dschönnseng“ die Ginseng-Wurzel zu erkennen, ist fast eine Denksportaufgabe! Die koreanische Bezeichnung „Insam“ scheint er nicht gehört zu haben.

Seine Vorstellung von der geistlosen Eintönigkeit buddhistischen Klosterlebens revidiert Genthe, nachdem er mit dem Abt des Klosters „Tschanganssa“ (Changan-sa) in den Diamantbergen (,Kimgangssan“ = Kumgang-san: heute in Nordkorea begrenzt zugängliche Touristenzone), der ihn gastlich aufgenommen hatte, philosophische Gespräche führen konnte. Lobend hebt er hervor, dass anders als in chinesischen Klöstern sanitäre Einrichtungen für Mönche und Gäste zur Verfügung standen. Uneingeschränktes Lob findet auch die landesübliche Fußbodenheizung, die den Koreanern - wiederum im Gegensatz zu Chinesen und Japanern - „,im Winter warme Stuben beschert“, allerdings um den Preis völliger Abholzung stadtnaher Wälder: Die später üblichen „Yongtan“-Briketts aus Kohlenstaub und Lehm waren offenbar noch nicht erfunden!

Manches bleibt allerdings auch für Genthe verwunderlich: Die Frauen, die in den Städten nach Sonnenuntergang zwei Stunden Ausgang haben, während die Männer zu Hause blei- 
ben müssen, verhüllen sich züchtig zu unförmigen Stoffbündeln, lassen aber die Brust frei, um ungehindert stillen zu können. Koreanische Reit- und Packpferde brauchen täglich eine warme Bohnensuppe als Mahlzeit - wonach sich die Reiseetappen zu richten haben - und würden bei Kaltfütterung angeblich eingehen. Ihre Artgenossen auf der Insel „Tschêdschu“ (Cheju) allerdings werden auch im Winter im Freien gehalten und müssen sich von der kargen kalten Weide nähren! Diese heute als Flitterwochenaufenthalt beliebte Insel war damals allerdings mangels Hafens schwer zugänglich und erscheint Genthe abweisend und bedrohlich, wohl schon durch ihre im Gegensatz zum landesüblichen Weiß ganz in Schwarz gekleideten Bewohner, die zudem kurz vor seinem Besuch über 300 Christen umgebracht hatten. Zweck seines Besuchs war die Erstbesteigung des die Insel beherrschenden Vulkankegels des „Hâlassan“ (Halla-san), die er auch erfolgreich durchführte: damals in wegelosem Gelände ein zweitägiges strapaziöses Abenteuer, heute eine eintägige, mäßig anstrengende Bergwanderung. Die Meereshöhe des Gipfels vermaß er korrekt mit $1950 \mathrm{~m}$.

So interessant all diese Schilderungen sind, eine Besprechung in VRÜ würden sie nicht rechtfertigen, wären da nicht die politischen und rechtlichen Betrachtungen, die sich immer wieder im Fluss der Erzählung finden. Das beginnt schon bei der Landung in Chemulpo mit dem Eindruck von der nicht nur optisch beherrschenden Stellung der Niederlassung der Hamburger Handelsfirma H.C. Eduard Meyer. Deren dort mit seiner Familie lebender Geschäftsführer Carl Wolter nahm den Besucher in sein gastfreies Haus auf. Er führte ihn auch in den „Tschemulpo-Klub“ ein, wo neben anderen Kaufleuten auch Angehörige der Zollverwaltung und Missionare verkehrten. Wirkungsvoll arbeitet Genthe den Kontrast zwischen dieser wohlhabend-gepflegten Umgebung und der mehr als bescheidenen Unterbringung des deutschen Konsuls in Seoul, Dr. Heinrich Weipert, in einem koreanischen Lehmgebäude heraus, während nicht nur Japan und die USA als Haupthandelspartner Koreas, sondern auch die europäischen Mächte Russland, Großbritannien, Frankreich, Italien und sogar Belgien ihre Vertretungen zu Gesandtschaften erhoben hatten, für die sie repräsentative Steinbauten errichteten. Da Konsul Weipert zudem als „Einzelkämpfer“ auftreten musste, denn die Vizekonsulsstelle war wenige Jahre zuvor einer Sparmaßnahme zum Opfer gefallen, spricht Genthe von einer „Aschenbrödelrolle“ Deutschlands, die den Koreanern einen völlig falschen Eindruck von dessen Bedeutung vermittle.

Ähnlich kritisch äußert sich Genthe zur mangelhaften finanziellen Förderung der deutschen Goldbergwerkskonzession „Tangkogä“ (Gonggogae) durch die deutschen Geldgeber: Die Unterkapitalisierung lasse eine erfolgreiche Prospektierung nicht zu.

Beeindruckt ist er dagegen von den Erfolgen der durch den ,wackeren Pommer“ Johannes Boljahn geleiteten staatlichen deutschen Sprachschule, von denen er sich durch Teilnahme an einer Abschlussprüfung überzeugen konnte.

In Seoul logierte Genthe gegenüber vom Palasteingang und hatte so einen optimalen Beobachterposten. Als erste Merkwürdigkeit registrierte er, dass die Staatsgeschäfte stets in der Zeit von Mitternacht bis zum Morgengrauen erledigt wurden - mit Anwesenheitspflicht für die Minister und Spitzenbeamten! Die vorangehenden Abendstunden gehörten meist 
Hoffesten im westlichen Stil, an denen er mehrfach teilnehmen konnte - arrangiert von der elsässischen „Haushofmeisterin“ Antoinette Sontag, einer Verwandten des russischen Gesandten C. v.Waebern. Einmal wurde Genthe in Privataudienz empfangen und konnte mit dem Kaiser (seit 1897, vorher König), dessen heute geläufiger Name „Kojong“ ihm nach alter Tradition erst nach Ende seiner Regierungszeit beigelegt wurde, ein längeres Gespräch führen. Er bekam den Eindruck eines wohlmeinenden und aufgeschlossenen, um das Wohl seines Landes und seiner Untertanen besorgten Herrschers, der sich eingehend nach den Eindrücken seines Besuchers aus dem Landesinneren erkundigte. Der ebenfalls anwesende Kronprinz machte ihm - wie sich später herausstellen solle, zu Recht - einen eher unbedarften Eindruck. Als Kuriosum merkt er an, dass mit jeder Postsendung aus Europa im Palast Briefe junger Damen (,Berliner Backfische“!) eingingen, die um Aufnahme in den kaiserlichen Harem baten...

Während seines durch ungünstiges Wetter unfreiwillig verlängerten Aufenthalts auf Cheju konnte Genthe ein fast freundschaftliches Verhältnis zum Gouverneur „I Dschä Ho“ (Yi $J a e-h o$ ) aufbauen und gewann so auch Einblick in die - mit der Verwaltung verbundene "Strafrechtspflege, die von früheren Autoren als „,noch grausamer als in China“ bezeichnet worden war. Nun lässt auch Genthe keinen Zweifel an der Grausamkeit der im auch in Korea geltenden „Ming-Kodex“ vorgesehenen Körperstrafen. Doch konnte er sich durch Augenschein überzeugen, dass die Folterwerkzeuge im Wortsinne eine Nummer kleiner waren als in China. Auch sei die bei Kapitalverbrechen vorgeschriebene Vierteilung in Korea stets nur an der Leiche des bereits Hingerichteten vollzogen worden, während in China der Delinquent bei lebendigem Leibe zerrissen wurde. Bei mehreren Besuchen im Gefängnis schienen ihm die in einer Gemeinschaftszelle untergebrachten Gefangenen obwohl an den Füßen angekettet - recht guter Dinge zu sein, mit Ausnahme einer zum Tode verurteilten Gattenmörderin, die auf einen astrologisch günstigen Termin für ihre Hinrichtung warten musste...

Soweit die Schilderungen. Von den dort wiederholt erwähnten fotografischen Aufnahmen Genthes konnte Bräsel ganze zwölf im von der „Library of Congress“ in Washington verwahrten Nachlass des Bruders Arnold Genthe ausfindig machen. Sie sind im Dokumentenanhang erstmals publiziert und lassen in Motivwahl und Qualität den Verlust des übergroßen Restes besonders bedauerlich erscheinen.

Wer sich von dem in Fraktur gedruckten Text abschrecken lässt, versäumt ein anregendes und lehrreiches Lesevergnügen.

Karl Leuteritz, Königswinter 


\section{Günter Hoog}

\section{Hamburgs Verfassung}

Aufriss, Entwicklung, Vergleich

Nomos Verlagsgesellschaft, Baden-Baden, 2004, 458 S., ISBN 3-8329-0931-1; 79,00 EUR

Hamburg als Handelsstadt war stets nach Übersee ausgerichtet. Im 19. Jahrhundert, während des Fehlens eines deutschen Zentralstaates, waren es die Stadtstaaten, die vor allem mit den neuen Staaten Lateinamerikas völkerrechtliche Verträge schlossen, so die gemeinschaftlichen Freundschafts-, Handels- und Schifffahrtsverträge von Lübeck, Bremen und Hamburg mit dem Kaiserreich Brasilien 1827, Venezuela 1831, Mexiko 1832 und Guatemala 1847, weiter die Verträge mit der Hohen Pforte 1839, mit dem Schah von Persien 1857 und der Vertrag Hamburgs und Lübecks mit dem Sultan von Sansibar. Hamburg selbst ist mit regelmäßig 90 Konsulaten drittgrößter Konsularplatz der Welt.

Wissenschaftlich war Hamburg mit der Kolonialzeit verbunden durch das am 20. Oktober 1908 eröffnete Kolonialinstitut, Vorgänger der am 10. Mai 1919 eröffneten Universität, die noch heute im Gebäude des vormaligen Instituts residiert. Schließlich sei auf den Internationalen Seegerichtshof hingewiesen.

Günter Hoog, ein exzellenter Kenner des öffentlichen Rechts, legt hier eine lehrbuchmäßige Darstellung von Hamburgs Verfassung von 1952 mit der Reform von 1996 vor. Angesichts der ungebrochenen Staatstradition wird die Verfassungsgeschichte vom Langen Rezess 1529 über den Hauptrezess 1712 (S. 64-68) bis zur Verfassung von 1860 (S. 68-70) dargestellt und die nachfolgende demokratische Verfassung von 1921 (S. 72 f.) als historisches Vorbild vergleichend herangezogen. So im 2. Kapitel (Schwerpunkt der Arbeit) über die Verfassung im Vergleich (S. 119 ff.) für die stadtstaatliche Einheitsgemeinde (S. 119133) oder das Deputationswesen (S. 181-187).

Hinzu tritt die intensive Rechtsvergleichung mit den Verfassungsgestaltungen in den norddeutschen Ländern, naturgemäß vor allem mit denen in den beiden anderen Stadtstaaten Bremen und Berlin. Diese von Hoog eröffnete Vergleichsebene ermöglicht es vermehrt, dass auch umgekehrt bei der Interpretation von Bremens und Berlins Verfassung auf die Hamburger Regelungen geblickt wird. Der Vergleich, er ist ein Markenzeichen dieser Arbeit, hebt die Besonderheiten in der Ausgestaltung der Hauptakteure des Verfassungslebens - Senat und Bürgerschaft - (S. 209 ff.) hervor. Bremen ist das einzige Land, das wie Hamburg, Art. 39 Verf., die Regelung einer Inkompatibilität zwischen Senatorenamt und Ausübung eines Bürgerschaftsmandates mit Ruhen dieses Amts kennt, Art. 108 Abs. 1 Verf. Bremen, S. 233. Nach dem Blick auf die historischen Regelungen seit 1712 (S. 234 f.) setzt sich Hoog ausführlich mit der Verfassungsmäßigkeit der Regelung in Art. 39 Verf. auseinander, die er bejaht, S. 239-248. Ausgangspunkt für seine Ausführungen ist das gegenteilige Urteil des hessischen Staatsgerichtshofes vom 7. Juni $1977^{1}$, welches indes selbst erwähnt, dass es in Hessen eine Inkompatibilität zwischen Regierungsamt und Abge-

1

Neue Juristische Wochenschrift (NJW) 1977, S. 2065 
ordnetenmandat nicht gibt. Hoog geht die einzelnen Bedenken durch, Verstoß gegen das parlamentarische Prinzip, den Grundsatz der Unmittelbarkeit der Wahl, Gleichheitssätze sowie gegen die Freiheit des Mandates?

Für die verfassungsmäßigen Aufgaben des Senats, S. 384 ff., stellt Hoog die Funktion als kollegiales Staatsoberhaupt voran, deutlich etwa in der Repräsentation nach außen oder in dem Begnadigungsrecht. Aus dieser Stellung wie auch aus der in Art. 33 Abs. 1 und 2, 42 Abs. 2 S. 2 Verf. festgelegten als Regierungskollegium ergibt sich der hervorgehobene Rang gegenüber dem einzelnen Senator und dem Ersten Bürgermeister. So dürfte auch nur das Kollegium und nicht der Erste Bürgermeister über Aufsichts- und Dienstaufsichtsbeschwerden gegen einen einzelnen Senator zu befinden haben.

Die Verfassungsreform vom 20. Juni 1996 hat die Stellung des Ersten Bürgermeisters über die bloße eines primus inter pares nach überkommenen Recht gestärkt : Er hat nicht nur den Stichentscheid bei Stimmengleichheit im Senat und das Recht, die Senatoren zu berufen und zu entlassen, sondern endlich auch die Richtlinienkompetenz, Art. 42 Abs. 2 S. 1 Verf. Hoog versteckt nicht seine Sympathie für das bisherige Kollegialsystem, indem er den Kritikern dieser Reform Raum gibt, einschließlich der überzogenen Kritik, dass die Verfassung eindeutig ,,autoritäre Züge“ erhalte (Hans Peter Ipsen), als ob in den anderen Ländern und im Bund autoritäre Strukturen vorherrschten. Die Lähmung in der senatsinternen Entscheidungsfindung hat der hamburgischen Politik insgesamt in der Vergangenheit nicht gut getan. Auf gewohnt sicherem juristischen Boden wird S. 388 ff. der Gegensatz behandelt, der sich ergibt, wenn einerseits in Angelegenheiten von grundsätzlicher oder allgemeiner Bedeutung der Senat als Kollegium zu entscheiden hat, Art. 42 Abs. 2 S. 2 Ziff. 4 Verf., andererseits der Erste Bürgermeister allein die Richtlinien der Politik bestimmt, die sich gerade in grundsätzlichen oder allgemeinen Fragen manifestieren wird. Ein Vorrang der Richtlinienkompetenz wie in Art. 65 S. 2 GG ist in Hamburg ausdrücklich nicht vorgesehen. Gleichwohl ist die erstmalige Einführung der Richtlinienkompetenz ein so gewichtiger Vorgang, dass die dadurch bewirkte zusätzliche Stärkung der Stellung des Ersten Bürgermeisters nicht durch den Rekurs auf Art. 42 Abs. 2 Verf. konterkariert werden darf und wird. In der politischen Praxis wird die Frage, ob der Erste Bürgermeister oder eine Fronde im Senat sich durchsetzt, nicht im Senat, sondern bei einer Einparteienregierung intern in der Regierungspartei und bei einer Koalitionsregierung zumeist durch den für eine Mehrheitsbildung benötigten kleineren Koalitionspartner entschieden.

Obwohl Hamburgs geltende Verfassung, anders als noch die von 1860 mit Einführung der Trennung von Staat und Kirche, keine Regelung des Verhältnisses von Staat und Kirche beinhaltet, geht Hoog auf dieses S. 38-43 ein. Das Staatskirchenrecht ist schließlich seit jeher Teil des Bundes- und Landesverfassungsrechts. Er stellt die Großkirchen und die anderen in Hamburg wirkenden Religionsgesellschaften mit öffentlich-rechtlichem Körperschaftsstatus vor einschließlich des ihnen verliehenen Kirchensteuerrechts, d.h. die Befugnis, die Mitgliedsbeiträge zwangsweise durchzusetzen. Inhaltliche Fragen, wie etwa die immer wieder streitigen zum Religionsunterricht in öffentlichen Schulen, Art. 7 Abs. 3 GG, sind inzwischen in den Staatskirchenverträgen vom 29. November 2005 geregelt, d.h. im 
Kirchenvertrag zwischen der Freien und Hansestadt Hamburg und der Nordelbischen Evangelisch-Lutherischen Kirche sowie im Vertrag zwischen dem Heiligen Stuhl und der Freien und Hansestadt Hamburg. Interessant ist dabei die Reihenfolge in der Erwähnung der Vertragspartner : Im evangelischen Kirchenvertrag schließt der Staat mit der auch in seinem Staatsgebiet ansässigen Landeskirche den Vertrag ab, im Konkordat ist es der die katholische Weltkirche repräsentierende Heilige Stuhl (und nicht das Erzbistum Hamburg), der einen Vertragsschluss gewährt (sublime Anwendung der kanonistischen Privilegientheorie?). Eine Hamburgensie besonderer Art enthält Art. 8 Abs. 5 des Kirchenvertrages: Danach verpflichtet sich die Nordelbische Evangelisch-Lutherische Kirche bei einem dringenden öffentlichen Bedarf Hamburgs an Grundstücken einer Kirchengemeinde, im Rahmen ihrer Verfassung darauf hinzuwirken, dass Hamburg diese Grundstücke, soweit sie nicht für kirchliche Zwecke benötigt werden, zu angemessenen Bedingungen erwerben kann. Diese lex Airbus ist Ergebnis dessen, dass im Streit um die Verlängerung der Startund Landebahn für die Luftwerft die evangelische Kirchengemeinde Hamburg-Neuenfelde ihre im Planungsgebiet liegenden Obstwiesen zunächst nicht veräußern wollte. Innerkirchliche Genehmigungsvorbehalte gelten nur für eine Veräußerung von Kirchengemeindegrundstücken und bedenken nicht den gegenteiligen Fall, dass eine Kirchengemeinde Grundstücke behalten will, um die Durchführung eines Planungsvorhabens zu durchkreuzen.

Wer eine gut lesbare und fundiert argumentative, zu allen aktuellen hamburgischen Verfassungsfragen Stellung nehmende Abhandlung sucht, wird mit dieser Arbeit bestens bedient. Ihre Anschaffung lohnt sich für Wissenschaftler wie Praktiker gleichermaßen.

Gerhard Scheffler, Hamburg

Matthias Basedau / Hanspeter Mattes / Anika Oettler (Hrsg.)

Multiple Unsicherheit. Befunde aus Asien, Nahost, Afrika und Lateinamerika

Schriften des Deutschen Übersee-Instituts Hamburg, Nr. 66

Hamburg, 2005, 266 S., 20,00 EUR, ISBN 3-926935-66-7

Sicherheit, persönliche zuvörderst, aber auch kollektive Sicherheit innerhalb einer lokalen oder regionalen Gemeinschaft, sodann nationale und schließlich internationale Sicherheit wohl in dieser Reihenfolge dürften die meisten Menschen hierzulande ihre Prioritäten zum Stichwort "Sicherheit" assoziieren. Der vorliegende Sammelband titelt allerdings von der negativen Seite her. Das ist konsequent, denn mit der Sicherheit ist es wie mit anderen existenziellen Gütern, Gesundheit etwa und Freiheit: Auch sie treten ins Bewusstsein erst bei Infragestellung, spätestens bei Verlust. Abzieherei auf dem Schulweg einerseits und 
kriegsanaloger Terrorismus auf der anderen Seite markieren die äußersten Pole im Spektrum solcher individuell wie kollektiv sicherheitszerstörenden Gewalterfahrung.

Diesem Spektrum sich widmend fußt der vorliegende Band auf einem Hamburger Kolloquium im Januar 2004 zum Thema "Innere Sicherheit/Sicherheit als öffentliches Gut in den Ländern des Südens“ innerhalb des Forschungsschwerpunkts "Gewaltdynamiken und Sicherheitskooperation", den das Deutsche Übersee-Institut derzeit unterhält. Die aus Referaten dieses Kolloquiums hervorgegangenen Beiträge von sieben Autoren und drei Autorinnen, allesamt Angehörige der fünf Hamburger Regional-Institute, welche den Verbund Deutsches Übersee-Institut bilden, zeichnen sich aus durch Kompaktheit und Prägnanz. Ganz offensichtlich war genügend Zeit, sich kurz zu fassen.

In ihrer gemeinsamen Einleitung ("Sicherheit in Asien, Nahost, Afrika und Lateinamerika Zum Forschungsgegenstand") umreißen die Herausgeber Dimensionen und Grenzen ihres Themas, dieses wiederum positiv gewendet: Sicherheit sei in vielen Ländern der untersuchten Regionen zum zentralen "unterproduzierten öffentlichen Gut" geworden, seit "Gewalt ... unterhalb und oberhalb der Kriegsschwelle, Staatszerfallsprozesse und Kriminalität ..." dort augenscheinlich eskalierten. "Äußere" Sicherheit sehen sie gewährleistet durch die "... Abwesenheit von Krieg ...". Das ist hinterfragbar, aber nicht eigentlich Gegenstand des Buches. Dessen Schwerpunkt liegt vielmehr auf der "Inneren" Sicherheit: Sie sei, so die Autoren, "... gewährleistet, wenn der Staat seine Bürger vor organisierter physischer Gewalt zu schützen und wenn ein unabhängiges Justiz- und ein gesetzestreues Sicherheitssystem Rechtsbrüche zu ahnden vermag". Ob ein solcher eher symptombezogener Begriffsansatz ungeteilten Beifall verdient, stehe dahin.

Im übrigen überrascht, wo so nachdrücklich von der Korrelation zwischen Unsicherheit und Staatsparalyse die Rede ist, dass ein spezifischer Beitrag zu den "failed states" fehlt. Bei näherer Betrachtung ist den Herausgebern allerdings Recht zu geben, wenn sie aus Raumgründen diese Thematik ausklammern und sich - von daher dankenswert - auf einen zentralen Literatur-Hinweis beschränken. ${ }^{1}$

Es sei im übrigen, so die Herausgeber, nicht ihr Bestreben, mit dem Sammelband eine Analyse vorzulegen, welche die dem Thema innewohnenden Fragen erschöpfe. Sie wollen vielmehr vom speziell regionalwissenschaftlichem Blickwinkel aus beitragen zum aktuellen Diskurs über Gewalt und Sicherheit, der in Deutschland nach ihrer Auffassung selten hinausreiche "... über den Tellerrand der westlichen Gesellschaften ...". Sie bezeichnen ihr Vorhaben als "... Bemühen, einen ersten Überblick zu geben und ausgesuchte Aspekte von Sicherheit in Afrika, Nahost, Asien und Lateinamerika zu beleuchten." Dieses Ziel hat der Band nicht verfehlt. Seine Beiträge tun genau dies: "beleuchten". Das impliziert notwendigerweise zweierlei: Zum einen, Dinge im Dunkeln zu lassen und zum andern, sie eben nicht zu durchleuchten. So ist beispielsweise immer wieder, aber eben auch nur beschreibend, 
die Rede von der Erosion staatlicher Gewaltmonopole, ohne das Gewaltmonopol, das ursprünglich ja ein Waffen-Monopol ist, auf seine Legitimität hin gleichermaßen zu befragen. Es dürfte wahrlich mancherorts staatliche Gewaltmonopole geben, deren baldiges Erodieren nur je wünschenswert wäre. Bisweilen fühlt man sich so erinnert an vergangene Zeiten, in denen die nicht legitimatorisch hinterfragte Vergötzung von "Stabilität" als Wert an sich und ohne Blick auf den Preis gang und gäbe war. Nur hin und wieder findet sich wenigstens die Frage nach den gesellschaftlichen und sozialen, aber auch namentlich religiös-fundamentalistischen wie kulturellen Ursachen von Sicherheitsdefiziten in den beobachteten Regionen.

Lassen wir dazu die Herausgeber selber zu Wort kommen: "... Gewalt hat dramatische soziale Folgen. Krankheiten und Seuchen finden einen idealen Nährboden in Bürgerkriegen. Gewalt führt zur Vernichtung von Sozialkapital. Gewalterfahrung zerstört wechselseitiges Vertrauen, das eine wichtige Entwicklungsressource ist. Die Alltäglichkeit von Gewalt produziert Gewöhnung und lässt Gewalt als eine akzeptable Form - oder Norm - der Lösung von Konflikten erscheinen. Darüber hinaus führen Konflikte zur Verbreitung von Kleinwaffen, die Gelegenheiten für Gewaltkriminalität dramatisch erhöhen. Gewaltsame Konflikte und weit verbreitete Gewaltkriminalität sind in ökonomischer Hinsicht ein erheblicher Standortnachteil. Humankapital wird ebenso vernichtet wie die Infrastruktur. Investoren werden durch eine prekäre Sicherheitslage abgeschreckt. Gewaltbelastete Gesellschaften leiden unter Kapitalflucht und ziehen allenfalls Risikoinvestoren an, die auf rücksichtlose Ausbeutung bzw. hohe Profitraten setzen und wenig Interesse an investitionsintensiven Projekten haben, die langfristig allen Beteiligten nützen. Wie erneut betont werden muss, sind Staatsschwäche oder Anomie nicht nur Ursachen, sondern direkt und indirekt auch Folgen von Gewalt und Kriminalität ..."(S. 26). Die Komplexität der Korrelation zwischen Gewalt und Entwicklung scheint schon in dieser Einleitung deutlich auf. In den Einzelbeiträgen findet sie ihre regionalen Entsprechungen.

Der erste Blick geht nach Asien. Marco Bünte ("Formen und Formenwandel politischer Gewalt in Südostasien - Ein Überblick") besticht mit einer plausibel aufbereiteten "Typologie politischer Gewalt". Ihre klassische Ur-Form zwischenstaatlicher Kriege allerdings sieht der Autor in der behandelten Region derzeit in den Hintergrund getreten zugunsten moderner Gewaltemanationen. Sie fächert er auf in Antiregimekonflikte, Sezessionistische Konflikte (z.B. Myanmar, Sumatra/Aceh, Philippinen/Mindanao, Sri Lanka/Jaffna) ${ }^{2}$ sowie die Konflikte aus transnationalen Komponenten des politischen Terrorismus (Abu Sayyaf, Laskar Jihad) und der internationalen Makro-Kriminalität (Drogen, Waffenschmuggel, Menschenhandel, Piraterie). Dass sich eine Vernetztheit dieser Gewaltfelder namentlich mit Blick auf den Al Quaida-Terrorismus empirisch nicht durchweg belegen lässt, wie der Autor aktuell resümiert, dürfte indes kaum genügen, um entsprechenden Befürchtungen für die Zukunft den Boden zu entziehen. 
Andreas Ufen, dieser Zeitschrift bereits als Autor verbunden ${ }^{3}$, konstatiert in seinem Beitrag ("Staatsschwäche als Erbe der Diktatur - Die Auflösung des militärisch dominierten Gewaltoligopols in Indonesien") eingangs Symptome zumindest partiellen Staatsversagens mit entsprechend destabilisierenden Konsequenzen für den nach der Entmachtung Suhartos 1998 hoffnungsvoll begonnenen Transitionsprozess: Zwar herrsche an Stelle des militärisch kontrollierten Gewaltoligopols der vormaligen "Neuen Ordnung" (1965-1998) inzwischen ein "... diffuses Gewaltoligopol mit einer Vielzahl unterschiedlicher Gewaltakteure in häufig undurchschaubaren Konstellationen ...". Das habe die Sicherheitslage wesentlich verschlechtert. Am Ende bleibt der Autor trotzdem verhalten optimistisch: Wenn auch der Staat auf einigen Feldern der inneren Sicherheit versage, drohe dennoch die Gefahr seines realen Zerfalls mittlerweile nicht mehr. Wichtigste Indizien hierfür: Landesweit halte sich die "... Politisierung von ethnischen und religiösen Konfliktlinien in Grenzen ...", habe bislang der Verfassungskompromiss gleichberechtigter Religionen im wesentlichen getragen und sei sogar die Restrukturierung der Streitkräfte befriedigend konfliktarm verlaufen.

Anschließend wendet sich der Band dem afrikanischen Kontinent zu. Hanspeter Mattes macht seine sehr eingängige Untersuchung ("Governing insecurity: Kriminalität und Kriminalitätsbekämpfung in Nordafrika") an bestimmten "Deliktslagebildern" fest. Sie lassen sich in geographischer Hinsicht nicht auf die nordafrikanischen Staaten begrenzen, müssen vielmehr auch den nördlichen Mittelmeerraum einbeziehen und zeigen mitunter sogar Auswirkungen bis nach Russland und in die Ukraine. In qualitativer Hinsicht überrascht kaum, dass sich regionsspezifische Delinquenz, wie sie signifikant vor allem in sozialen Disparitäten wurzelt, mittlerweile eher weniger im politischen Terrorismus (Stichwort: Algerien), als in gesteigerter allgemeiner Kriminalität niederschlägt, bemerkenswert umfangreich mittlerweile in transnational organisierter Form. Gesellschaftliche Gegenreaktionen (Anti-Gewalt-Demonstrationen, zivilgesellschaftlicher Konsens, Bürgerwehren) korrespondieren, so der Autor, mit vergleichbar ermutigenden Ansätzen auf staatlicher Ebene, die etlichenorts im nordafrikanischen Raum zu greifen begännen: Anti-Korruptionskampagnen, Reorganisation der Polizeistrukturen, verbesserte technische Ausstattung, Strafrechtsanpassungen, Präventionskonzepte, sonstige Umfeldreformen. Fortschritte mache neben der besonders mit den UN und Interpol gepflegten polizeilichen Kooperation auch die intraregionale Kooperation. Sie findet sich institutionalisiert etwa im schon 1982 gegründeten Rat der arabischen Innenminister (RAIM/CAMI) mit Sitz in Tunis und den seinem Generalsekretariat zugeordneten speziellen Dependancen in Bagdad, Damaskus, Amman, Casablanca und Kairo unter wissenschaftlicher Begleitung durch die Naif Arab Academy for Security Sciences (NAASS) in Riad. Mit welchen längerfristigen Erfolgsaussichten sich in den Spannungsfeldern zwischen sozioökonomischen Rahmenbedingungen, staatlichen Reformstrategien, negativen Globalisierungseffekten und transnational gene- 
rierten Profiten der organisierten Kriminalität trotzdem politisch allgemeinwohlorientiert manövrieren lässt, muss der Autor notgedrungen offen lassen.

Martin Beck ("Sicherheit im Vorderen Orient") beleuchtet Dimensionen und Perspektiven einer "Sicherheit auf orientalisch". Den schon geographischen Ausmaßen seines Themas immerhin von Casablanca bis zum Hindukusch - Rechnung tragend beschränkt er sich auf zwei Thesen: Die eine, empirisch begründete, geht dahin, dem orientalischen Staat ein vergleichsweise unangefochtenes Gewaltmonopol zu attestieren: Privatisierte Gewalt und damit einhergehender sicherheitspolitischer Kontrollverlust des Staates im Vorderen Orient konstituierten im Vergleich mit anderen Entwicklungsregionen keinen dominanten Trend. Die andere, normative These - und insoweit wird hier die Legitimitätsfrage gestreift - geht dahin, die Kehrseite des sicherheitsstiftenden Gewaltmonopols zu artikulieren: Seine Instrumentalisierung zum Niederhalten zivilgesellschaftlicher Opposition unabhängig von deren angeblichen Nähe zur politischen Gewaltbereitschaft. Seine Thesen evaluiert der Autor mit differenzierten Ergebnissen an den Beispielen Algerien, Sudan, Palästina, Libanon, Jemen, Irak und Afghanistan.

Manches aus diesen Überlegungen kehrt wieder im ausgesprochen lesenswerten Beitrag von Henner Fürtig ("Innere Sicherheit in arabischen Kernstaaten - Die islamistische Herausforderung"). Auf die Beispiele Ägypten und Saudi-Arabien beschränkt, wird die innere Sicherheit im Blick auf das nicht ernsthaft in Frage gestellte staatliche Gewaltmonopol weniger aus der Perspektive "von unten" (als Sicherheit der Bürger vor Kriminalität), sondern aus der Perspektive "von oben" (als Eigensicherung des autoritären Staates) untersucht. Überzeugend wird mit eingehendem Blick auf die ägyptische Muslimbruderschaft und ihre traditionell reformislamische Opposition ebenso wie auf die die reine Lehre hütende Opposition in Saudi-Arabien plädiert für eine redliche Differenzierung zwischen Islamismus und Terrorismus, ohne vor aktuellen Schnittmengen die Augen zu verschließen. Was Mitherausgeber Matthias Basedau ("Sicherheitsprobleme im subsaharischen Afrika Ein Überblick") zu vielfach verbreiteten Sicherheitsdefiziten in den 48 Staaten seiner ins Auge gefassten Region beizutragen hat, lässt mittelfristig nicht mehr ruhig schlafen. Es geht im Grunde nur darum, hier Sicherheit als höchst relativen Begriff zu definieren. Damit wird Sicherheit zur Ausnahmeerscheinung, was es wiederum rechtfertigt, für diese Region Unsicherheit in so gut wie allen Beispielen als Regelfall zu konstatieren.

Andreas Mehler ("Gewaltoligopole" und "Sicherheit als kollektives Gut" - Konzeptionelle Überlegungen mit Illustrationen aus Westafrika) geht sein Thema vom ökonomischen Blickwinkel in marktwissenschaftlicher Terminologie an. Er startet mit der ohne weiteres plausiblen Feststellung, der europäisch geprägte Staatsgedanke mit Fiskalhoheit und legitimem Gewaltmonopol habe in Westafrika wenig Realität, sei von den Amtsinhabern höchst unvollkommen verinnerlicht, bestehe gleichwohl als Anspruch der Eliten fort, weiche realiter allerdings Gewaltoligopolen, "...die der Bevölkerung oder Teilen derselben einen variierenden Grad an Erwartungssicherheit sowie variierende Orientierungsrahmen bieten." Unter "Gewaltoligopol" versteht er "... eine Aufteilung der Gewaltmittel auf wenige Gewaltakteure bzw. "Schutzgeber, die voraussichtlich überwiegend partikulare Zielsetzun- 
gen verfolgen". Das schmeckt nach Mafia und Triaden, setzt sich auch so fort in den Erklärungsmustern vom "Sicherheitsmarkt", von "Sicherheit-Nachfrage"-Strukturen und vom "Angebot der Schutzgeber und Sicherheitserzeuger". Eine für Nicht-Ökonomen zunächst gewöhnungsbedürftige, aber im Ergebnis dann doch faszinierende Perspektive.

Sabine Kurtenbach ("Gewalt, Kriminalität und Krieg in Lateinamerika - Zur symbiotischen Verbindung verschiedener Gewaltformen und den Problemen ihrer Einhegung") untersucht Verbindungen und Verknüpfungen der verschiedenen Gewaltphänomene nach Organisationsform (individuelle versus kollektive Gewalt) und - wie sie es nennt - "Legitimation" von Gewalt (politisch versus sozioökonomisch). Namentlich mit den Beispielen Kolumbien und Guatemala illustriert sie, wie sich kriminelle und politische Gewaltstrukturen verzahnen in Anpassung an durchaus wechselnde politische Kontexte. Ihr Fazit zur Einhegung dieser hochexplosiven Mischung bezieht überzeugend auch die Notwendigkeit ein, die Erwerbschancen einer inzwischen besser ausgebildeten und mit höherer Lebenserwartung ausgestatteten, auch hinreichend leistungsbereiten Jugend in Lateinamerika zu forcieren.

Zum Interessantesten, was der Band insbesondere für Angehörige der juristischen Zunft zu bieten hat, gehört der Beitrag von Anika Oettler ("Lynchjustiz in Guatemala. Produktion und Erosion von Sicherheit"). Nach jahrzehntelangem auf-sich-gestellt-sein beim Erleiden bewaffneter Gewalt, gleichgültig ob herrührend von sogenannten "Sicherheitsorganen", paramilitärischen oder revolutionären Kräften, kehren die dörflichen Gemeinschaften zurück zu vorkolonialen Mechanismen der Selbsthilfe bei der Wahrung ihrer unmittelbaren lokalen Sicherheit, primär vor alläglicher Kleinkriminalität, mitunter aber auch vor schwerer wiegender Delinquenz: "Opfer solcher Lynchjustiz", so die Autorin, "... waren entweder die Anführer von Verbrecher- oder Jugendbanden, die zuvor die Bevölkerung - unbehelligt von Polizei und Justiz - in Angst und Schrecken versetzt hatten, oder es handelte sich um Kleinkriminelle, die beim Stehlen (u.a. von Heiligenbildern) auf frischer Tat ertappt worden waren ...". Rechtsstaatlich-institutionelle Postulate wie etwa Rechtsgleichheit, Richtervorbehalt, Gesetzesvorrang, Unschuldsvermutung, verhallen hier ungehört, weil in jahrzehntelanger alltäglicher Gewalterfahrung empirisch diskreditiert. Das lässt die Länge und Strapazen des Weges erahnen, der zurückzulegen ist, bis von einem - freilich nur demokratisch legitimierbaren - Gewaltmonopol des Staates die Rede sein kann.

Bedauerlicherweise nur kurz schlussendlich dasjenige, was Ulrike Borchardt gleichsam als Ausblick schildert ("Möglichkeiten und Grenzen der 'Security Sector Reform'"). Eine "... effektive zivile Kontrolle über die Reform des Sicherheitssektors, d.h. in Sicherheits- und Verteidigungsangelegenheiten kompetente Parlamentarier, die Einbeziehung der Zivilgesellschaft in die Reform, ein offener Konsultationsprozess zwischen Militär, Parlamentariern und Zivilgesellschaft ...", das sind für sie unabdingbare Voraussetzungen für eine wirksame Reform des Sicherheitssektors. Dem zunächst unbefangen nickenden Publikum muss sich bei näherem Hinsehen allerdings die Frage aufdrängen, was diese idealtypischen "Voraussetzungen" ihrerseits wiederum an Voraussetzungen voraussetzen, damit ein solcher Reformprozess überhaupt in Gang kommen kann. Internationale Hilfestellung, wie 
die Autorin sie abschließend fordert, vermag die notwendige interne Initialzündung nicht zu ersetzen.

Schade, dass man dieses Buch nur ein einziges Mal lesen kann. Anschließend zerfällt es nämlich in seine Einzelteile. Dies bei dem nicht unerheblichen Preis an die Adresse des Verlages.

Karl-Andreas Hernekamp, Hamburg 